\title{
Primary leaflet tear without dystrophic calcifications in a 25 years old Ionescu-Shiley pericardial valve in mitral position
}

\author{
Astrid Quessard ${ }^{1}$, Nadjib Hammoudi ${ }^{2}$, Matthias Kirsch ${ }^{* 3}$ \\ ${ }^{1}$ Department of Anaesthesiology, Assistance Publique - Hôpitaux de Paris, Groupe Hospitalier Pitié Salpêtrière, Institut de \\ Cardiologie, Université Pierre et Marie Curie, Paris, France \\ ${ }^{2}$ Department of Cardiology, Assistance Publique - Hôpitaux de Paris, Groupe Hospitalier Pitié Salpêtrière, Institut de \\ Cardiologie, Université Pierre et Marie Curie, Paris, France \\ ${ }^{3}$ Department of Cardiac Surgery, Assistance Publique - Hôpitaux de Paris, Groupe Hospitalier Pitié Salpêtrière, Institut de \\ Cardiologie, Université Pierre et Marie Curie, Paris, France
}

Received: June 9, 2015

DOI: $10.5430 /$ css.v1n $1 \mathrm{p} 8$
Accepted: July 1, 2015

URL: http://dx.doi.org/10.5430/css.v1n1p8

\begin{abstract}
Despite its excellent hemodynamic performance, the Ionescu-Shiley bovine pericardial bioprosthesis was removed from clinical use in 1987 because of an unacceptable high incidence of primary structural deterioration of the valve, mainly related to its design. Few examples of very long-term durability of this prosthesis have been recently reported. In all these cases, the valve dysfunction was caused by primary severe dystrophic calcification and/or pannus formation. In contrast, we describe the unexpected 25-year longevity of a mitral Ionescu-Shiley bioprosthesis which failed due to primary tears of one cusp but without dystrophic changes.
\end{abstract}

Key Words: Ionescu-Shiley pericardial valve, Bioprosthesis, Long-term durability

\section{INTRODUCTION}

Despite excellent hemodynamics, the Ionescu-Shiley bovine pericardial bioprosthesis was withdrawn from clinical use because of a high incidence of structural deterioration. We describe the unexpected 25-year longevity of a mitral IonescuShiley bioprosthesis which failed due to primary tears of one cusp but without dystrophic changes.

\section{CASE REPORT}

In June 1986 a 45-year-old man underwent mitral valve replacement with a 27-mm Intact porcine bioprosthetic valve (Medtronic, Minneapolis, Minn.) for rheumatic mitral dys- function (patient unable to take anticoagulants). Eight months later, due to an early non-structural prosthetic dysfunction, a new mitral valve replacement with a $27-\mathrm{mm}$ lowprofile Ionescu-Shiley pericardial bovine bioprosthesis (Shiley Laboratory, Irvine, CA) was performed.

The patient did well for 25 years. In February 2012, he presented to the emergency department with symptoms of decompensated congestive heart failure. Physical examination revealed a mitral holosystolic murmur and pulmonary bilateral basal crackles. There was no clinical or biological sign of infection. A two-dimensional echocardiographic study

\footnotetext{
*Correspondence: Matthias Kirsch; Email: matthias.kirsch@bch.aphp.fr; Address: Department of Thoracic and Cardiovascular Surgery, Cardiology Institute, La Pitiè-Salpêtrière Hospital, 47-83 Bd de l'Hôpital, Paris, France.
} 
showed severe eccentric intra prosthetic mitral regurgitation due to the prolapse of one bioprosthetic cusp (see Figures 1 and 2) but no other associated abnormality was detected: all 3 cusps were thin without calcification and no vegetation was detected. The left ventricle was not dilated and showed a slightly impaired systolic function (45\%). Pulmonary arterial pressure was measured at $55 \mathrm{mmHg}$.

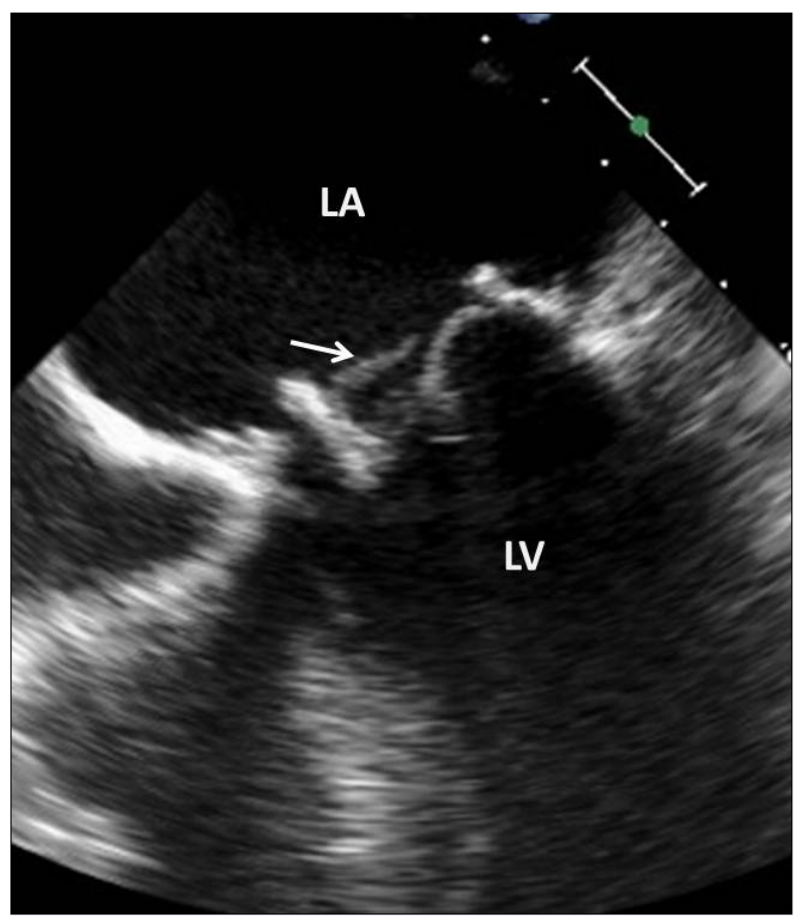

Figure 1. Trans oesophageal echocardiography $\left(4^{\circ}\right)$ in systole: One cusp prolapse of bioprosthetic mitral valve (arrow). Note the absence of cusp thickening or calcification. LA: left atrium, LV: left ventricle.

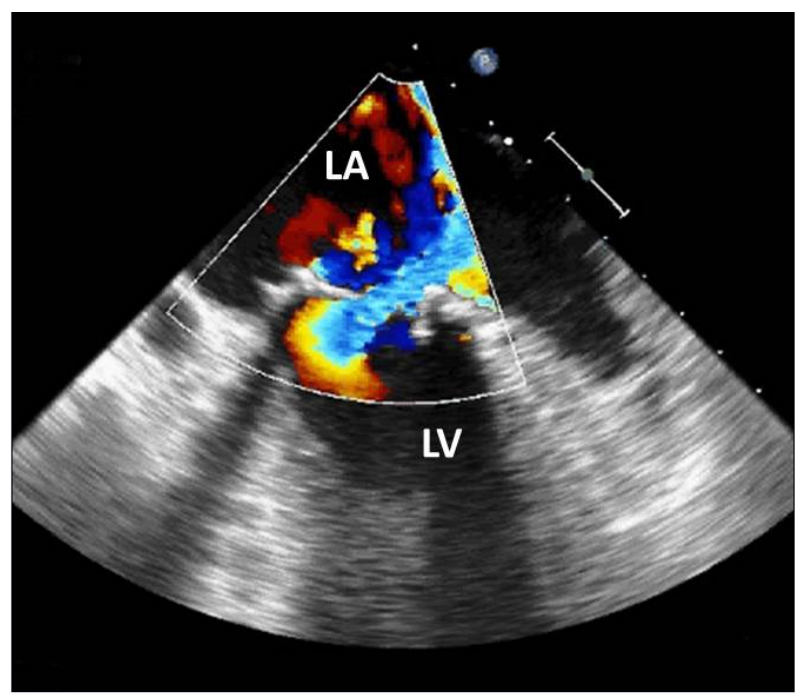

Figure 2. Trans oesophageal echocardiography $\left(62^{\circ}\right)$ with color Doppler: Severe eccentric mitral regurgitation
After successful medical treatment for heart failure and exclusion of coronary artery disease, the patient underwent replacement of the Ionescu-Shiley valve with a 27-mm CarpentierEdwards Perimount Magna mitral heart valve (Edwards Lifesciences Corp., Irvine, CA) in April 2012. Macroscopic examination of the explanted prosthesis revealed no cusp calcification or pannus formation, but 2 tears, respectively 6 $\mathrm{mm}$ and $3 \mathrm{~mm}$ deep, were observed on one leaflet at the site of its attachment to the commissural stent posts (see Figure 3 ). Furthermore, no vegetations were noted and cultures of the valve remained negative.

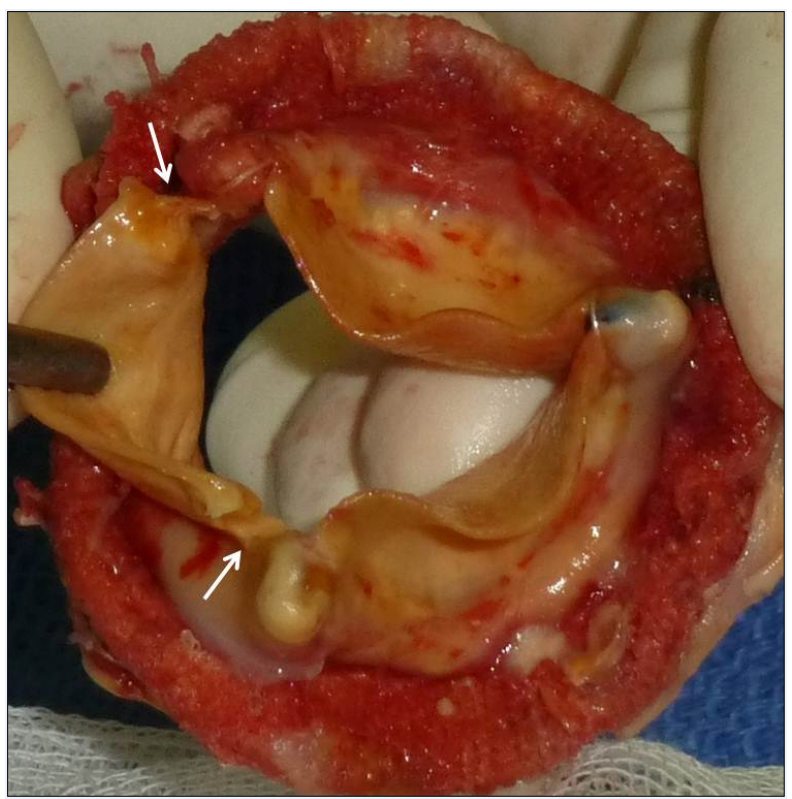

Figure 3. Operative specimen: 2 tears (arrows) on one leaflet at the site of its attachment to the commissural stent. No cusp thickening, no calcification.

The postoperative course was complicated by sepsis related to central catheter and surgical wound infections. The patient was discharged from hospital in a stable condition 2 months after surgery. Echocardiographic examination showed mild impairment of left ventricular function and normal bioprosthetic valve function.

\section{Discussion}

The Ionescu-Shiley bioprosthetic valve (ISBP) was developed in 1971 and represents the first generation xenograft made from bovine pericardium. When compared to porcine bioprostheses, this valve showed excellent hemodynamic performances because of the absence of the muscle shelf at the base of the valve and greater symmetrical opening of all 3 valve leaflets resulting in a larger effective orifice area. However, an unacceptable high incidence of primary structural deterioration of the valve led to its withdrawal from clinical 
use in 1987. This was related to valve design rather than to the physico-chemical and biological properties of bovine pericardium. Tears at or close to the "alignment stitches" that hold the cusps to the stents represented the most common form of early tissue valve failure. These changes occurred earlier with low-profile ISBP than with the standard-profile model, in both aortic and mitral positions.

In cases where the valve was removed more than 5 years after implantation, a lower rate of cusp tears was reported and degenerative processes such as cusp calcification and tissue overgrowth were the predominant causes of valve dysfunction. ${ }^{[1]}$ Some examples of extreme durability of the ISBP have been published recently both in aortic ${ }^{[2,3]}$ and mitral positions. $^{[4,5]}$ However, in these reports, late valve failure was primarily related to severe dystrophic changes of the valve leaflets with major calcifications eventually leading to secondary tears and/or significant pannus formation. In contrast, the ISBP we retrieved after 26 years in mitral position failed due to primary tears of one cusp but without dystrophic calcification and no significant pannus overgrowth.

Thus, our observation illustrates the potential for long-term sustainability of pericardial tissue as a heart valve substitute, even when implanted in young recipients. In the future, improved understanding of pathogenesis of structural valve deterioration with better identification of valve- and host related risk factors will allow the development of preventive measures, the design of even more durable bioprostheses, and, eventually, their use in younger recipients.

\section{REFERENCES}

[1] Walley VM, Keon CA, Khalili M, et al. Ionescu-Shiley valve failure. II: Experience with 25 low-profile explants. Ann Thorac Surg. 1992; 54: 117-22. http://dx.doi.org/10.1016/0003-4 975 (92) $91155-3$

[2] Yankah CA, Weng Y, Meyer R, et al. Twenty-two-year durability of Ionescu-Shiley pericardial aortic bioprosthesis implanted in a 49year-old woman: a valuable insight into the performance of current pericardial bioprostheses. J Thorac Cardiovasc Surg. 2006; 132: $427-$ 8. PMid: 16872976. http://dx.doi.org/10.1016/j.jtcvs.2 006.04 .031
[3] Honda K, Okamura Y, Nishimura Y, et al. Twenty-six-year durability of an Ionescu-Shiley standard profile pericardial aortic valve. Interact Cardiovascular Thoracic Surg. 2010; 10: 315-316. PMid: 19914926 http://dx.doi.org/10.1510/icvts.2009.210898

[4] Watanabe U, Ohkawa S, Yamabuki K. A case of 24 years longevity of an Ionescu-Shiley bioprosthesis in the mitral position. Asian Cardiovasc Thorac Ann. 2006; 14: 96-98. http://dx.doi.org/10. $1177 / 021849230601400529$

[5] Fiore A, Cooley DA, Grande AM, et al. Unusual 25-year durability of an Ionescu-Shiley pericardial bioprosthesis. Ann Thorac Surg. 2011; 91: e52-3. PMid: 21440107. http://dx.doi.org/10.1016/j.a thoracsur.2010.11.019 